\title{
RELATO DE EXPERIÊNCIA DA ORGANIZAÇÃO DE UM EVENTO SOBRE FISSURA LABIOPALATINA POR UMA LIGA ACADÊMICA DE CIRURGIA PLÁSTICA DE FORTALEZA
}

\author{
Laís Fabrício de Oliveira Cunha \\ Universidade Estadual do Ceará \\ laisfabricioc@gmail.com \\ Laís Simões Teixeira \\ Universidade Estadual do Ceará \\ laisteixeira@hotmail.com
}

\begin{abstract}
Resumo
$\mathrm{O}$ trabalho aqui desenvolvido apresenta-se como um relato de experiênciadirecionado a organização de um evento realizado pela Liga de Cirurgia Plásticada Universidade Estadual do Ceará (LCP-UECE) abordando o tema de fissura labiopalatina. Evidencia inicialmente, uma abordagem teórica sobre a fissura labiopalatina, bem como a apresentação da liga responsável pelo evento. No que diz respeito ao relato propriamente dito, foramexplicitadas as características do evento bemcomo o questionário que foi aplicado na ocasião, assim como seu objetivo, que foi de descobrir o quê e quanto a população sabe realmente sobre a fissura labiopalatina para assim evidenciar a importância desse tema para a sociedade, prestando os devidos esclarecimentos após a aplicação, quando necessário. Ao fim da intervenção foi possível concluir que existe uma necessidade de divulgar mais o tema, assim como alertar para a sua importância e informar a população na intenção de desmistificar algumas crenças, que se mostraram presentes no momento da intervenção.
\end{abstract}

Palavras-chave: Fissura labiopalatina. Cirurgia plástica. Medicina.

\section{EXPERIENCE REPORT OF THE ORGANIZATION OF EVENT ABOUT LABIOPALATIN FISSURE BY ACADEMIC LEAGUE OF FORTALEZA PLASTIC SURGERY}

\begin{abstract}
This work is presented as an experience report directed to the organization of an event held by the League of Plastic Surgeryof the State Universityof Ceará (LCP-UECE) addressing the topic of cleft lip and palate. It initially shows a theoretical approach to cleft lip and palate, with the introduction of it, as well as the presentation of the league responsible for the event. Regarding the report itself, the characteristics of the event were explained, as well as the questionnaire that was applied at the time, as well its objective, which was to find out what and how much the population really knows about the cleft lip and palate in order to evidence the importance of this topic to society, providing due clarifications after implementation, when necessary. At the end of the intervention it was possible to conclude that there is a need to disseminate the theme further, as well as to alert its importance and inform the population in the intention to demystify some beliefs, which were present at the time of the intervention.
\end{abstract}

Key words: Cleft lip. Palate. Plastic surgery. Medicine.

\section{RELATO DE EXPERIENCIA DE LA ORGANIZACIÓN DE UM EVENTO SOBRE FISURA LABIOPALATINA POR UNA LIGA ACADÉMICA DE CIRURGÍA PLÁSTICA DE FORTALEZA.}

\section{Resumen:}

El trabajo aquí desarrollado se presenta como un relato de experiencia dirigido a la organización 
de un evento realizado por la Liga de Cirugía Plásticade la Universidad Estatal de Ceará (LCPUECE) abordando el tema de fisura labiopalatina. Evidencia inicialmente, un enfoque teórico sobre la fisura labiopalatina, así como la presentaciónde la aleaciónresponsable del evento. En lo que se refiere al relato propiamente dicho, se explicita las características del evento así como el cuestionario que se aplicó en la ocasión, así como su objetivo, que fue de descubrir qué y cuánto la población conoce realmente sobre la fisura labiopalatina para así evidenciar la importancia del tema para la sociedad, prestando las debidas aclaraciones después de la aplicación, cuando sea necesario. Al final de la intervención fue posible concluir que existe una necesidad de divulgar más el tema, así como alertar para su importancia e informar a la población con la intención de desmitificar algunas creencias, que se mostraron presentes en el momento de la intervención.

Palavras clave: Fisura labiopalatina. Cirurgía plástica. Medicina. 


\section{INTRODUÇÃO}

A experiência das Ligas como fenômeno de articulação e mobilização de indivíduos ou grupos em torno de uma necessidade ou carência remonta à Antiguidade, quando as Ligas de Delos e do Peloponeso, em um contexto de guerra entre Atenas e Esparta nas disputas por território, buscavam desenvolver práticas educativas articuladas à estratégia de defesa (SILVA; FLORES, 2015).

No campo da saúde, as Ligas emergiram da necessidade de combater a alta prevalência de agravos à saúde pela tuberculose e pela hanseníase no início do século XX, período de transição de uma economia agroexportadora para uma economia que rumava para a industrialização e urbanização do Brasil. Essas Ligas Acadêmicas eram formadas por voluntários das elites intelectuais e da Faculdade de Medicina da Universidade de São Paulo - USP (SILVA; FLORES, 2015).

Segundo Hamamoto Filho (2011), foi na USP que nasceu a primeira Liga brasileira, criada na década de 1920. Era a Liga de Combate à Sífilis, criada por estudantes com o intuito de intervir médica e socialmente num problema de saúde pública da época. A ausência do Estado brasileiro no campo da saúde pública à época foideterminante para que esse tipo de ação fosse adotada (SILVA; FLORES, 2015).

Ainda de acordo com Hamamoto Filho (2011), a partir da década de 1990, a quantidade de Ligas criadas no Brasil cresceu rapidamente, coincidindo com períodos de reformas curriculares e intenso debate político e acadêmico a respeito do perfil do médico a ser formado. Também houve aumento expressivo de Ligas Acadêmicas em outras áreas da saúde, como nos cursos de Enfermagem, mas a maioria destas organizações ainda se concentra nos cursos de Medicina (SILVA; FLORES, 2015).

As Ligas Acadêmicas são entidades formadas por grupos de alunos de diferentes anos da graduação sob a supervisão de profissionais e professores vinculados a Instituição de Ensino Superior ou Hospitais de Ensino e propõem-se a fomentar o desenvolvimento do ensino e da pesquisa, proporcionando um rico cenário de práticas aos estudantes, que têm a oportunidade de estabelecer vínculos com professores e com a comunidade. Tal proposta possibilita ao aluno de graduação uma maior proximidade com temas e conhecimentos de especialidades médicas, sendo as ligas acadêmicas um importante recurso na integração da atividade médica e científica (SILVA; FLORES, 2015).

De acordo com Monteiro et al. (2008), os princípios básicos que norteiam essas entidades são atividades de pesquisa, ensino e assistência e estão contidos em estatutos. Além disso, os estatutos também estabelecem: a denominação, a sede, a finalidade e o modo de funcionamento da Liga; os requisitos de admissão e exclusão e os direitos e 
deveres dos membros; a forma de gestão administrativa; as condições para disposições regimentais e dissolução da Liga (SILVA; FLORES, 2015).

Compreendidas no rol de possibilidades de currículo paralelo, assumindo um caráter extracurricular e complementar, as Ligas têm se configurado como parte do cotidiano dos estudantes, desde o ingresso no curso médico. Nas Ligas, os discentes desenvolvem atividades teóricas (aulas, seminários, análise e discussão de textos e apresentações de casos clínicos), organizam cursos, simpósios e congressos, atuam em projetos de pesquisa, participamde atividades de assistência médica em cenários diversos e tomam parte de campanhas e eventos públicos de promoção à saúde. As atividades práticas, por sua vez, são desenvolvidas em hospitais, instituições filantrópicas filiadas e unidades básicas de saúde (HAMAMOTO FILHO, 2011; SILVA;FLORES,2015).

Nesse contexto, e sob as mesmas premissas, a Liga de Cirurgia Plástica da Universidade Estadual do Ceará (LCP UECE) foi criada em 2009 por acadêmicos de Medicina de períodos variados da Universidade Estadual do Ceará (UECE), que se reuniram com a ideia comum de fundar um grupo de estudos que pudesse discutir e elucidar temas em Cirurgia Plástica Estética e Reconstrutiva, e, mais do que isso, praticá- los.

Trata-se de uma entidade sem fins lucrativos, com duração ilimitada e de caráter multidisciplinar, regida pelos princípios de ensino, pesquisa e extensão. É um projeto vinculado ao Centro de Ciências da Saúde da UECE, ao Conselho de Ligas Acadêmicas da UECE e à Associação Brasileira das Ligas de Cirurgia Plástica (ABLCP).

A Liga é atualmente composta por dezessete membros (dez membros da graduação, quatro residentes e três professores ou médicos assistentes da UECE), tem como finalidade proporcionar aproximação entre graduandos e a cirurgia plástica, uma vez que a maioria dos estudantes desconhece muitos dos campos de atuação desta especialidade, tais como: queimaduras, cirurgia crânio-maxilo-facial, cirurgia de mão, mama, abdome e cuidados de ferimentos complexos; bastante frequentes na prática médica. O acesso à LCP UECE é realizado por meio de processo seletivo (prova e entrevista) após curso introdutório realizado anualmente.

As reuniões da Liga de Cirurgia Plástica da UECE são realizadas quinzenalmente, momento no qual os participantes ministram seminários abertos a todos acadêmicos do curso de Medicina da UECE, mesmo que não sejam esses ligantes. A LCP UECE proporciona, ainda, aos membros a possibilidade de estágio no serviço de residência em Cirurgia Plástica do Hospital Universitário Walter Cantídeo (HUWC), acompanhando os procedimentos cirúrgicos de grande porte e ambulatoriais do HUWC e do Instituto José 
Frota (IJF).

A extensão é um dos três pilares básicos das instituições universitárias, sendo esses pilares: ensino, pesquisa e extensão. Através da extensão os estudantes universitários podem contribuir para melhoria de aspectos necessários a sociedade, aplicando diretamente aquilo que é desenvolvido dentro dos outros dois pilares, ensino e pesquisa. Dessa forma, sob uma ótica assistencialista, os acadêmicos podem prestar um serviço à população voltada para suas necessidades (JEZINE, 2004). Foi regido por esse pilar da extensão que se realizou a intervenção dos ligantes da LCP UECE, com a intenção de compreender e suprir a necessidade da população de conhecimento sobre a fissura labiopalatina.

Levando em conta o que foi exposto, o presente o relato consiste na experiência de uma extensão realizada na cidade de Fortaleza, pela Liga de Cirurgia Plástica da Universidade Estadual do Ceará (LCP UECE), destacando sua organização e execução. Seu objetivo é evidenciar as conclusões obtidas com a experiência da intervenção com a população fortalezense, bem como relatar os incentivos e obstáculos encontrados na abordagem do tema escolhido.

\section{DESENVOLVIMENTO}

Em Maio 2017, a LCP foi convidada para participar de um evento sobre saúde na Praça Nossa Senhora de Fátima, localizada na Avenida 13 de Maio, bairro de Fátima, no município de Fortaleza, em comemoração ao Dia das Mães. Para isso, deveria escolher um tema de saúde pública relacionado com a Cirurgia Plástica para abordar com os participantes do evento.

O tema escolhido foi Fissura Labiopalatina. O evento ocorreu no dia 06 de Maio de 2017, aberto ao público e contou com a prestação de variados serviços de saúde à população distribuídos em tendas. A participação da LCP aconteceu por meio da abordagem dos indivíduos presentes para explicações e esclarecimentos acerca do tema escolhido. Conforme experiências anteriores, os ligantes já sabiam que a tenda precisaria dispor de algum serviço mais atrativo para facilitar a captação de ouvintes. Dessa forma, levaram um esfigmomanômetro e um estetoscópio para verificar a pressão daqueles que desejassem. De fato, a oferta desse serviço fez, notoriamente, com que muitas pessoas procurassem a tenda da LCP. Após a aferição da pressão, cada cidadão era convidado a responder um questionário que continha perguntas elaboradas pelos ligantes sobre conhecimentos básicos acerca do tópico a ser abordado na oportunidade. Não era 
necessário que as pessoas se identificassem e também não eram obrigadas a responder se não quisessem.

O objetivo desse questionário era descobrir o quê e quanto as pessoas sabem sobre o assunto para entender se o tema realmente é relevante para a população, se ela precisa de maiores esclarecimentos, e, assim, melhorar nossas extensões e produzir pesquisa naquilo que os respondedores mostrassem deficiência.

Com relação às explanações, elas foram realizadas com auxílio de banner ilustrado confeccionado pelos ligantes, que mostrava as informações de maneira didática. Cada exposição durava cerca de 10 minutos, e os ligantes (7 estavam presentes na ocasião) revezavam-se nas apresentações.

O conteúdo das explanações se referia à definição de fissura labiopalatina e orientações básicas sobre as alterações anatômicas, o desenvolvimento do paciente fissurado e como alimentar corretamente o fissurado, incluindo aleitamento materno.

Com relação à definição, as fissuras labiopalatinas são definidas como malformações congênitas que ocorrem durante a gestação, no período embrionário, entre a quarta e a nona semana. Essas malformações se devem a fatores genéticos, agentes tóxicos, agentes infecciosos, estresse, radiações ionizantes e uso de algumas drogas aos quais as gestantes são submetidas, por ação isolada ou em associação (SILVA; FÚRIA; DI NINNO, 2005; DA SILVA; SANTOS, 2004).

O componente genético é muito importante dadas as evidências de elevada porcentagem, mais de 50\%, de que fissurados tenham membros da família que também são portadores da síndrome. Os fatores ambientais podem ser os mais diversos, desde estresse até tabagismo (SILVA; FÚRIA; DI NINNO, 2005). Essas evidências demonstram a importância de uma coleta de história familiar bem-feita e completa, bem como questionamento sobre fatores socioambientais, que muito influenciam na existência desses casos.

É estimado que a incidência de casos mundialmente seja em torno de 1 a cada 600700 nascimentos (BILINSKA; OSMOLA, 2015). No Brasil, não há ao certo uma estimativa da prevalência e da incidência, já que os estudos geralmente não incluem as regiões Norte e Nordeste. Entretanto, segundo a Organização Mundial de Saúde (OMS), há cerca de 300 mil casos de brasileiros portadores de fissuras labiopalatinas, uma a cada 650 nascimentos, dado que converge com o índice mundial (RIBEIRO; MOREIRA, 2005). Esses dados demonstram a importância de se falar com a população sobre o assunto, fazendo-a perceber que não é uma doença rara, tampouco distante da realidade de todos. 
No que diz respeito às alterações anatômicas, foi incluído na explicação a classificação de Spinna et al. (1979), em: fissura pré-forame incisivo completa e incompleta, unilateral, bilateral ou mediana; fissura transforame incisivo (unilateral, bilateral, mediana); fissura pós-forame incisivo (completa ou incompleta) e fissuras raras da face (apud BATISTA; TRICHES; MOREIRA, 2011; CYMROT, et al. 2001).

No que se refere ao desenvolvimento do paciente fissurado, colocou-se para os presentes o que Da Silva e Santos (2004) evidenciam em seu estudo: a fissura labiopalatina é o principal fator de problemas na funcionalidade de estruturas anatômicas relacionadas a fala. A maioria dos pacientes fissurados apresenta problemas para falar de maneira inteligível e, dessa maneira, para realizar efetivamente a comunicação verbal e oral.

Para pacientes com fissura apenas labial, pode-se enfatizar as dificuldades de sucção e deglutição, além das dificuldades típicas da fala, como a produção de alguns fonemas específicos. Isso se justifica pelo fato de que os lábios são intimamente envolvidos na articulação e manutenção da pressão da cavidade oral para produção de alguns fonemas, podendo o paciente produzi-los de maneira alterada ou podendo esses fonemas estarem até mesmo ausentes (DA SILVA; SANTOS, 2004).

Diante das várias dificuldades estruturais, funcionais e estéticas que o paciente enfrenta, é fato, relatado em literatura e percebido na prática médica diária, que esses indivíduos têm o seu desenvolvimento psicossocial também prejudicado, enfrentando preconceito e rejeição tanto de seus familiares como da sociedade.

Dessa forma, se fazia relevante explicar para a população que as modificações estéticas causadas por esta sequela, muitas vezes, geram nos indivíduos afetados: baixa autoestima, evasão de contatos sociais, medo, vergonha, insegurança, ansiedade, depressão, inibição dependendo do grau de deformidade e estresse. Tais fatores, associados à dificuldade comunicativa, podem interferir na funcionalidade do indivíduo, prejudicando sua inclusão social (BACKES et al., 2012). Segundo Murray et al. (2010), pesquisas têm apontado para uma diminuição da competência social de crianças com fissura labiopalatina, que se caracterizam pelo menor número de amigos, menos contatos sociais e pior qualidade de interações sociais.

Assim, a qualidade de vida e inclusão social desses pacientes estão extensamente conectadas com a efetividade do tratamento, cirúrgico e não cirúrgico. De acordo com Veronez (2007), mesmo quando tratadas, esses pacientes, muitas vezes, permanecem com dificuldades de comunicação, impactando no julgamento que o indivíduo faz de si mesmo e no julgamento dos outros pela sua aparência ou déficit funcional, o que 
repercute nos seus relacionamentos afetivos, estudo e trabalho.

Quanto à alimentação, foi explicitado no momento da intervenção que existem divergências sobre qual o melhor método a ser adotado, como em questões de posicionamento ou o tipo de alimentação, por exemplo. Mas o leite materno é comumente recomendado, não apenas pelo seu valor nutricional, importante para o recém-nascido, mas pelo seu poder antibacteriano, que pode auxiliar na defesa de infecções que são comuns aos recém-nascidos fissurados, como as infecções da orelha média. (SILVA; FÚRIA; DI NINNO, 2005).

Em adição ao fator nutricional, a amamentação como ato apresenta grande relevância para o desenvolvimento da criança fissurada, pois auxilia de maneira importante no seu desenvolvimento facial. Sem contar que os reflexos da sucção e deglutição estão preservados nesses pacientes, mesmo que essas ações sejamdificultadas pela sua alteração anatômica (BATISTA; TRICHES; MOREIRA, 2011).

Todas essas informações foram repassadas durante o evento que teve duração de aproximadamente $8 \mathrm{~h}$ e contou com a participação e atenção, efetivamente, de 150 pessoas dentre todas as que participaram do evento.

Desde sua fundação, a LCP UECE realizou inúmeras extensões com o intuito de informar e esclarecer a população sobre Fissura Labiopalatina, bem como outros temas que fazem parte dos projetos da Liga, mas essa foi, sem dúvida, a de maior público e de maior duração.

\section{CONCLUSÃO}

O trabalho em equipe possibilitou a organização de um evento da área de saúde de grande relevância para o bairro. Tal iniciativa auxiliou a difundir o tema, alertar para sua importância e desmistificar certas crenças. Além disso, ficou evidente para os acadêmicos que a população em geral ainda é bastante leiga no que diz respeito a esse assunto, sendo relevante que eventos como esse continuem a acontecer. Com a prestação do serviço de aferição de pressão, confirmou-se, mais uma vez, que as pessoas ainda temem algumas doenças específicas, como hipertensão e diabetes, crendo que somente elas são realmente muito prejudiciais e precisam de atenção e cuidado, e ignoram completamente - algumas pessoas sequer já tinham ouvido falar sobre o tema abordado outras tantas doenças importantes. Algumas pessoas não quiseram ouvir a explanação, aferiram a pressão e deixaram a tenda. Conclui-se, assim, que o graduando em Medicina, 
por meio das ligas, tem um importante papel social, visto que tem a oportunidade de conversar mais de perto com a população, de esclarecer dúvidas individuais e, assim, contribuir para a disseminação do conhecimento e, possivelmente, fazer a diferença na vida de alguém, já que as informações obtidas poderão ser necessárias a qualquer momento para qualquer cidadão. 


\section{REFERÊNCIAS}

BACKES, D. S., BACKES, M. S., Erdmann, A. L., \& Büscher, A. O papel profissional do enfermeiro no Sistema Único de Saúde: da saúde comunitária à estratégia de saúde da família. Ciência \& Saúde Coletiva, v.17, n.1, p.223-230, 2012.

BATISTA, L. R. V., TRICHES, T. C., \& MOREIRA, E. A. M. (2011). Desenvolvimento bucal e aleitamento materno em crianças com fissura labiopalatal. Revista Paulista de Pediatria, v.29, n.4, p.674-679.

BILINSKA, M.; OSMOLA, K. Cleft lip and palate -risk factors, prenatal diagnosis and health consequences. Ginekol Pol, v. 86, n. 11, 2015.

CYMROT, M., SALES, F., TEIXEIRA, F., JUNIOR, F., TEIXEIRA, G., \& OLIVEIRA, N. Prevalência dos tipos de fissura em pacientes com fissuras labiopalatinas atendidos em um Hospital Pediátrico do Nordeste brasileiro. Revista Brasileira de Cirurgia Plástica, v.25, n.4, p.648-651, 2001.

DA SILVA, R. N.; \& SANTOS, E. M. N. G. Ocorrência de alterações da motricidade oral e fala em indivíduos portadores de fissuras labiopalatinas. Revista Brasileira em Promoção da Saúde, v.17, n.1, 2004.

DA SILVA, E. B.; FÚRIA, C. L. B.; DI NINNO, C. Q. M. S. Aleitamento materno em recém-nascidos portadores de fissura labiopalatina: dificuldades e métodos utilizados. Revista CEFAC, v. 7, n. 1, 2005.

DA SILVA, S. A.; FLORES, O. Ligas Acadêmicas no Processo de Formação dos Estudantes. Rev. Bras. Educ. Med., Rio de Janeiro, v.39, n.3, p.410-417, 2015.

HAMAMOTO FILHO, P. T. Ligas Acadêmicas: motivações e críticas a propósito de um repensar necessário. Revista Brasileira de Educação Médica, [s.1.], v. 35, n. 4, p.535543, dez. 2011.

JEZINE, E. As práticas curriculares e a extensão universitária. In: Congresso Brasileiro de Extensão Universitária. p. 1-5, 2004.

MONTEIRO, L. L. F.; CUNHA, M. S.; OLIVEIRA, W. L.; BANDEIRA, N. G.; MENESES, J. V. Ligas acadêmicas: o que há de positivo? Experiência de implantação da Liga Baiana de Cirurgia Plástica. Rev. Bras. Cir. Plást. v.23, n.3, p. 158-161, 2008.

MURRAY, L., ARTECHE, A., BINGLEY, C., HENTGES, F., BISHOP, D. H., GOODACRE, T.; HILL, J. The effect of cleft on sócio-emotional functioning in schoolaged children. Journal of Child Psychology and Psychiatry and Allied Disciplines , v.51, n.1, p. 94-103, 2010.

RIBEIRO, E. M.; MOREIRA, A. S. C. G. Atualização sobre o tratamento multidisciplinar das fissuras labiais e palatinas. Revista Brasileira em Promoção da Saúde , v. 18, n. 1, p. 31-40, 2005.

VERONEZ, F. de S. Avaliação da Qualidade de Vida em Pacientes Adultos com Fissura Labiopalatina. Dissertação (Mestrado) - Hospital de Reabilitação de Anomalias Craniofaciais da Universidade de São Paulo, 2007. 